\title{
Limitaciones para el control de la corrupción en las acciones de interdicción de drogas en el VRAEM+
}

\author{
FÁTIMA ROJAS* \\ Pontificia Universidad Católica del Perú \\ fatima.rojasb@pucp.edu.pe \\ https://doi.org/10.18800/rcpg.201601.003
}

\begin{abstract}
RESUMEN
En un escenario como el de los valle de los Ríos Apurímac, Ene y Mantaro (VRAEM), con presencia de actividades vinculadas al narcotráfico, se busca evadir el control estatal para la continuidad del negocio criminal mediante prácticas de corrupción. Y la policía, responsable de los operativos de interdicción de drogas, es un actor susceptible a este fenómeno. Ante ello, se han generado medidas para el control de las prácticas de corrupción policial; no obstante, existen limitaciones para una implementación efectiva. En esta investigación se sugiere que las limitaciones responden a: i)la disociación entre el tipo de medida anticorrupción y el tipo de práctica de corrupción; ii) la disposición y gestión de recursos y iii) el modo de coordinación desde el nivel sub-nacional que reduce la capacidad de respuesta del funcionario policial a nivel local para corrupción en la interdicción de drogas.
\end{abstract}

Palabras clave: capacidades estatales, corrupción, policía, política de drogas.

\section{Limitations for the control of corruption in drug interdiction actions in the Vraem}

\begin{abstract}
In a scenario such as the Apurímac, Ene and Mantaro Rivers Valley (VRAEM), with presence of activities related to drug trafficking, it seeks to evade state control for the continuity of the criminal business through corruption practices and the police, responsible of drug interdiction operations, is an actor susceptible to this phenomenon. Before this, measures have been taken to control police corruption practices; However, there are limitations to effective implementation. This research suggests that limitations: (i) the dissociation between the type of anticorruption measure and the type of corruption practice; (ii) the provision and management of resources; and (iii) the mode of coordination from the sub-national level that reduces the police officer's ability to respond at the place for corruption in drug interdiction.

Key words: state capacities, corruption, police, drug policy.

\footnotetext{
* Investigadora adjunta del Laboratorio de Criminología de la PUCP. El presente artículo ha sido elaborado tomando como base la tesis de Maestría en Ciencia Política titulada: Una lectura a las limitaciones burocráticas para el control de la corrupción en las acciones de interdicción de drogas en el VRAEM. El documento ha sido elaborado en el marco del Programa de Apoyo a la Investigación para estudiantes de Posgrado (PAIP) y para la edición de la Revista de Ciencia Política y Gobierno de la Escuela de Gobierno y Políticas Públicas.

+ Recibido el 12 de abril de 2016; aceptado 26 de septiembre de 2016.
} 



\section{INTRODUCCIÓN}

La implementación de las medidas de control de la corrupción en un escenario de convivencia con el narcotráfico es un desafío para el Estado peruano. La disposición de recursos y tácticas de violencia en el primer valle cocalero del Perú ha llevado a que los operadores de seguridad, como la Policía Nacional, intervengan mediante operativos de interdicción con el objetivo de detectar producción de derivados cocaínicos y los insumos para su producción, así como capturar y detener a los agentes involucrados en el transporte del producto. Sin embargo, con la existencia del tráfico ilícito de drogas en la zona, se ha desarrollado prácticas de corrupción que buscan perpetuar el negocio ilícito. En esta investigación analizaremos las dificultades que tienen las fuerzas policiales para hacer frente a este fenómeno de la corrupción que contrapone los esfuerzos de la lucha contra las drogas.

La presente investigación plantea como estudio de caso el despliegue de medidas anticorrupción en el marco de las acciones de interdicción policial de derivados cocaínicos en el valle de los ríos Apurímac, Ene y Mantaro (VRAEM). ${ }^{1}$ El citado valle constituye la principal zona de producción de droga, puesto que el escaso acceso a servicios públicos y los problemas de seguridad han generado cierta dependencia económica al cultivo de coca que es destinada a la producción de drogas cocaínicas (UNODC 2014, IDEI 2009).

En este escenario, a través de los operativos de interdicción de drogas, los policías, como representantes del Estado, tienen el deber de identificar, perseguir y eliminar el desvío de derivados cocaínicos, por lo que su labor no puede verse interrumpida por prácticas de corrupción que benefician a intereses privados. Por lo tanto, el presente estudio pretende abordar cómo se desarrolla el control de las prácticas de corrupción que se interfieren en las acciones de interdicción de drogas cocaínicas en el VRAEM. En ese sentido, el objetivo de investigación es comprender el control de las prácticas de corrupción en los operativos antidrogas en el valle del VRAEM por parte de la Policía Nacional.

Para tal fin, se proponen como objetivos específicos: i) describir las prácticas de corrupción que suceden en los operativos de interdicción de drogas en el VRAEM, ii) determinar las limitaciones de las medidas anticorrupción para el control de las acciones de interdicción de drogas en el VRAEM, y iii) explicar las causas de las limitaciones para la implementación de las medidas

\footnotetext{
De acuerdo al decreto supremo $\mathrm{N}^{\circ}$ 021-2008 se determinan los distritos que forman parte del esquema de intervención estratégica denominado "Plan VRAE» y mediante el decreto supremo Nº40-2016-PCM se determina el reordenamiento de los ámbitos de intervención directa del VRAEM para llegar de un modo propicio e integral a la población habitante de estos territorios.
} 
anticorrupción para el control de las acciones de interdicción de drogas en el VRAEM.

De manera preliminar, se sostienen tres hipótesis construidas en relación a cada uno de los objetivos indicados: en primer lugar, que las prácticas de corrupción tienen un grado de ocurrencia según el margen de maniobra del funcionario policial en el marco de los operativos de interdicción de drogas. En segundo lugar, si bien existen medidas anticorrupción, se cree que estas presentan dificultades en su implementación, como 1) disposición y gestión de recurso, 2) ineficacia administrativa, 3) disposición y gestión de logística y 4) organización para la realización de sus funciones. Finalmente, como tercera hipótesis, se cree que las causas de las limitaciones para la implementación de las medidas anticorrupción están asociadas a 1) la disociación de la medida con el tipo de fenómeno a combatir/prevenir la corrupción por no entender el fenómeno y 2) esta disociación entre la medida y el tipo de fenómeno genera concentración y sobrecarga laboral, lo que devendría en un déficit de elementos burocráticos como la gestión y distribución del recurso humano y logístico, organización de la estrategia anticorrupción, entre otros. Ahí se cree que el elemento burocrático destaca las características, entre las cuales está la estabilidad en el cargo y la capacidad técnica para el desarrollo de las labores, sobre todo en zonas a nivel subnacional como es el VRAEM.

A continuación se exponen las premisas teóricas que acompañan la investigación, seguido por el planteamiento metodológico del estudio. Asimismo, se presentarán los hallazgos organizados en: i) las prácticas de corrupción que se manifiestan en el VRAEM, ii) las limitaciones que tienen las medidas anticorrupción es este valle y iii) las causas que explicarían las limitaciones identificadas. Finalmente, se discuten algunos elementos teóricos pertinentes a partir de los hallazgos que se presentan a modo de conclusión.

\section{CAPACIDADES ESTATALES PARA LA LUCHA CONTRA LA CORRUPCIÓN EN MATERIA DE TRÁFICO ILÍCITO DE DROGAS: ESTUDIOS, DEBATES Y DEFINICIONES}

Los estudios desde la ciencia política sobre el fenómeno de corrupción lo relacionan con el sistema político. Así, por ejemplo, se han buscado las causas de la corrupción debatiendo sobre mayor o menor presencia del fenómeno en un autoritarismo o una democracia (Colazingari \& Rose-Ackerman 1998, Seligson 2002, Canache \& Allison 2005), en un sistema democrático descentralizado o no (Weyland 1998, Manor 1999, Shleifer \& Vishny 1993, Wilson 1989a), responsabilizándolo de la falla de fórmulas de repartición del poder en democracia como la rendición de cuentas, transparencia y 
procedimientos formales de gobernabilidad (Doig \& Theobald 2000), así como por el fracaso del liderazgo ético (Hope 2000: 19), entre otros enfoques. Sin embargo, dentro de la literatura en ciencia política existe un consenso por definir la corrupción como la desviación de las actividades normales propias de la función pública en búsqueda de satisfacer bienes privados, económicos, de estatus (Theobald 1999, Rose-Ackerman 2001, Sampford 2006), o cuando se violan las reglas contra el ejercicio de ciertos tipos de influencia privada (Nye 1967). Algunos otros autores especifican el mal uso de la función pública (Johnston 1996, Treisman 2000) o el desvío de sus funciones institucionales (LaPalombara 1994). Es decir, se tiene una noción de corrupción como el abuso de un determinado poder a partir de una función pública, lo cual corresponde al ámbito del Estado.

En ese sentido, la ciencia política también otorga un espacio para explicar la relación entre la corrupción y la burocracia, de modo que puede acercarnos a una definición más precisa y de interés para este estudio. De acuerdo a Mauro (1995), cuando las burocracias dilatan las decisiones, muchas veces los actores privados se ven en la necesidad de otorgar incentivos ilegales para acelerar los trámites. No obstante, es preciso indicar que la burocracia está compuesta por más elementos que son importantes considerar. Dentro de la organización burocrática existen niveles de funcionamiento y a partir de ellos es necesario identificar el tipo de corrupción. Mientras la corrupción política o grand corruption política toma lugar en los niveles de autoridad y de poder de decisión (jefes o cabeza de Estado, ministerios y ejecutivos) (Moody-Stuart 1997), la corrupción burocrática, o petty corruption, es la corrupción que se realiza en la administración pública, en la implementación de las políticas (Alfiler 1979); es decir, los agentes adecúan las modalidades de corrupción al sistema de operaciones de la burocracia. Por su lado, Mujica (2011) considera que todas las actividades de la micropolítica, por más pequeñas que sean, van a convertirse en actividad política que reinventa el poder y sus estructuras de acción. En esa línea, tomando en cuenta que existen niveles de funcionamiento en el sistema burocrático y que en estos espacios puede encontrarse un tipo de práctica de corrupción, siguiendo a Mujica (2011), esta investigación entiende la corrupción como un fenómeno fragmentario; es decir, a partir de cómo se manifiesta en la realidad.

Ahora, la corrupción en el sector policial refiere a todo aquello que el policía hace o deja de hacer en contra de su responsabilidad por alguna ganancia material, financiera o la promesa de dicha ganancia (Punch 2009), es decir, todo tipo de conducta para obtener un beneficio en virtud de la posición oficial 
del policía (Roebuck y Barker 1974: 424), que al ser «agentes de ley», su función es cumplirla y no ir en contra de la misma (Melville Dalton, 1959). Además, la motivación de la conducta corrupta en el policía puede ser para obtener un logro o ascenso personal (Perez \& Moore 2002, Ivkovich 2005: 549). Pues bien, las variaciones en las definiciones de corrupción dentro de la literatura también se pueden encontrar por examinar los tipos de comportamientos que han sido categorizados en diversas tipologías. Las tipologías de corrupción policial muchas veces están catalogadas como más o menos graves en función de quién está involucrado, normas o leyes quebradas, el apoyo entre pares, cómo se organiza el hecho, la reacción de la oficina policial (las sanciones internas) y las motivaciones subyacentes del actor (Barker y Roebuck 1973), todo ello es un elemento importante que luego, en nuestro caso de estudio, permite comprender la situación de los policías que incurrieron en una práctica de corrupción.

Para establecer tipos de corrupción policial se establecen grados, desde las formas menos graves de corrupción, como cualquier corrupción de la autoridad que pide o recibe gratificaciones o comisiones, hasta las formas más graves de corrupción, tales como el robo oportunista, extorsiones, la protección de las actividades ilegales o comportamiento criminal directo (Barker y Roebuck 1973). Sumado a ello, Punch (2009) indica una categoría determinada para definir las conductas de corrupción policial en caso del narcotráfico que consiste en generar o adicionar pruebas para culpar a alguien y asegurarse de tener una larga sentencia criminal.

Tomando en cuenta lo mencionado sobre la corrupción como fenómeno fragmentario y el caso de estas prácticas en el sector policial, en esta investigación se entiende que las medidas anticorrupción deberían tener un diseño que responda a la configuración del fenómeno de corrupción en la realidad operativa del policía. Sin embargo, las medidas anticorrupción no solo podrían tener problemas de diseño, sino también problemas durante la implementación, y por ello los funcionarios operativos requieren de ciertas capacidades para poder abordar estas prácticas y poder responder adecuadamente a las medidas anticorrupción.

La noción de capacidad (estatal) es pertinente para comprender cómo finalmente el Estado, en tanto una burocracia profesional, es capaz de implementar una política sin influencia externa indebida (Bersch, Praça \& Taylor 2013). En ese sentido, las capacidades burocráticas refieren a los mecanismos de identificación y control de los espacios de autonomía y a las actividades para modificar la rutina, generando alternativas que evidencien la 
intención oficial de la política (Lattuada \& Nogueira 2011). Particularmente, en esta investigación nos concentramos en el tipo de capacidad que refiere a la habilidad del Estado, o de una institución específica de este, para hacer efectiva la entrega de bienes y servicios y la capacidad de planificación, determinación y control de los procesos y rutinas durante la implementación de la política (Sikkink 1993). Es decir, toda actividad que desarrolla la burocracia bajo una organización y autoridad para obtener sus objetivos (Jacob 1966; Weber, 1946). Ello nos lleva a considerar la capacidad burocrática como la habilidad para que los funcionarios o burócratas sean capaces de desarrollar la política debido a su comportamiento, a los recursos y estrategias que emplean para ejecutar sus actividades en función de la política (Barzelay 2001, Repetto 2004, Cortázar 2007).

Además, considerando el funcionamiento de la burocracia, en esta investigación nos centramos en el funcionamiento operativo, las actividades desarrolladas y ejecutadas en la práctica por los funcionarios, pues nos pueden decir más de los resultados que las reglas promulgadas formalmente (Peters, 1999). Ahora, dado que el proceso de implementación de políticas públicas es una fuente de acción e inacción, no se manifiesta en una sola acción y varía constantemente (Hill \& James 1993), existen dos instancias que la literatura reconoce como las principales para abordar el análisis de implementación de la política pública. Algunos investigadores adoptan un enfoque topdown o "de arriba hacia abajo» que se concentra en los aspectos del proceso de implementación a partir de quienes deciden formalmente la política y pueden controlarla (Matland 1995, Van Meter \& Van Horn 1975); mientras que otros autores resaltan el enfoque teórico-práctico bottom-up o «de abajo hacia arriba» y enfatizan los grupos objetivos con el argumento de que la política se hace realmente desde abajo, es decir, se identifica con la posición de quienes son considerados como los «funcionarios públicos locales» (Streetlevel bureaucrats) (Lipsky 1971), es decir, aquellos funcionarios que interactúan directamente con los ciudadanos. A los funcionarios locales se les asigna tareas y por sus condiciones de trabajo están expuestos a cambios constantes y deben acomodarse a las demandas de la realidad como: recursos limitados, imprecisión de fines, la presión político-administrativa, entre otros (Aguilar 1993). En otras palabras, los funcionarios públicos locales se adecúan a las circunstancias y "procuran desarrollar prácticas que les permitan procesar el trabajo que se les exige» (Lipsky 1971), por ello, para efectos de una mejor comprensión de las capacidades estatales-burocráticas en la implementación de 
medidas en el nivel subnacional, como es el espacio denominado VRAEM, es pertinente considerar las características de este actor.

Con estas consideraciones conceptuales desarrollaremos un análisis del funcionamiento de la administración del Estado desde una institución particular como la Policía Nacional respecto a los casos de corrupción en los operativos antidrogas y las medidas que se toman para el control de estas prácticas irregulares. Por lo tanto, en el desarrollo de los capítulos se detallará la conducta que las medidas anticorrupción apuntan a controlar durante la implementación de los operativos de interdicción de drogas, así como las actividades concretas que la Policía Nacional emplea con la finalidad de ejercer control sobre las prácticas de corrupción.

\section{Metodología}

El estudio tiene corte cualitativo, y plantea un análisis descriptivo y explicativo del fenómeno. Con la finalidad de describir procedimientos operativos, identificar patrones y explicar las limitaciones, se emplearon entrevistas semiestructuradas a los actores pertinentes: policías que trabajan en Inspectoría (tanto de Inspectoría General como del VRAEM), en las unidades policiales del VRAEM, en la Dirección Anticorrupción, así como procuradores anticorrupción y especialistas en materia de tráfico ilícito de drogas. Asimismo, se revisaron documentos administrativos que destacan la revisión de expedientes de Inspectoría sobre los casos de corrupción policial, así como información periodística relevante al tema de estudio. Como se señala a continuación, se exploraron tres tipos de lugares ricos en datos, para los cuales se dispone una serie específica de herramientas e instrumentos. 


\section{Tabla 1. Lugares ricos en datos, herramientas e instrumentos para el recojo de información}

\begin{tabular}{|c|c|c|}
\hline Lugares ricos en datos & Herramientas & Instrumentos \\
\hline $\begin{array}{l}\text { Bibliografía, normativa, } \\
\text { documentos administrativos }\end{array}$ & $\begin{array}{l}\text { Revisión de archivo } \\
\text { cualitativo }\end{array}$ & Ficha de revisión bibliográfica \\
\hline Información de prensa & $\begin{array}{l}\text { Revisión de archivo } \\
\text { periodístico }\end{array}$ & Inventario de noticias \\
\hline \multirow{3}{*}{$\begin{array}{l}\text { Experiencia de expertos, } \\
\text { funcionarios, servidores y } \\
\text { actores locales }\end{array}$} & $\begin{array}{l}\text { Entrevistas en } \\
\text { profundidad }\end{array}$ & $\begin{array}{l}\text { Guía de entrevistas para entrevistas a } \\
\text { especialistas en Tráfico Ilícito de Drogas }\end{array}$ \\
\hline & & $\begin{array}{c}\text { Guía de entrevistas a funcionarios en } \\
\text { Tráfico Ilícito de Drogas }\end{array}$ \\
\hline & & $\begin{array}{c}\text { Guía de entrevistas a servidores de segu- } \\
\text { ridad en Tráfico Ilícito de Drogas }\end{array}$ \\
\hline
\end{tabular}

En relación a lo expuesto en la tabla, se revisó y clasificó la información bibliográfica y de prensa según los objetivos de investigación. Además, se revisaron resoluciones del tribunal de disciplina policial entre los ańos 2013 y 2014 y se sistematizó cada expediente según sanción, retiro, absolución de la sentencia y nulidad de la sentencia de acuerdo a las medidas Muy Graves vinculadas al tráfico ilícito de drogas y la «solicitud o recibimiento de dádivas o beneficios». ${ }^{2}$ Se aplicaron quince entrevistas entre especialistas con experiencia académica sobre el tráfico ilícito de drogas, funcionarios, responsables de jefatura o con cargo ejecutivo en la Policía Nacional como en la Procuraduría Anticorrupción en casos relativos a la policía, así como a servidores o funcionarios operativos que laboran en inspectorías como en las unidades policiales locales en el VRAEM.

El estudio ha implicado la aplicación de criterios éticos y de seguridad, acordes a lo previsto por el Comité de Ética para la Investigación con Seres Humanos y Animales de la PUCP. ${ }^{3}$ Ello ha significado el uso de protocolos de consentimiento informado para los participantes, así como medidas de cuidado

\footnotetext{
2 Las faltas Muy Graves consideradas fueron: MG-27 (DL-1150) y MG-30 (DL-29356): «Participar, favorecer o facilitar actividades ilícitas relacionadas a tráfico ilícito de drogas, terrorismo, lavado de activos, secuestro, trata de personas, espionaje, robos, violación, extorsión y otros delitos graves"; así como MG-49 (DL-1150) y MG-62 (DL29356): «solicitar o recibir dádivas o cualquier otra clase de beneficio proveniente directa o indirectamente del usuario del servicio o de cualquier persona que tenga interés en el resultado de su gestión».

3 Para mayor información se puede revisar la página web de la Oficina de Ética de la Investigación e Integridad Científica (OETIIC) en la bibliografía.
} 
y autocuidado del investigador. De la misma manera, se han considerado criterios de reserva de la identidad y de custodia de la información sensible.

\section{Hallazgos del estudio}

\section{Principales prácticas de corrupción en los operativos de interdicción de drogas en el VRAEM}

Actualmente, el VRAEM cuenta con el 19\% de la superficie cultivada y el 54\% de la producción potencial de cocaína (UNODC 2014). En esta realidad hay presencia de cultivo de hoja de coca, pozas y laboratorios para la producción de derivados cocaínicos, así como acopio y transporte (terrestre y aéreo) de los mismos. En este espacio existe disposición de recursos y condiciones geográficas, y la población en las condiciones mencionadas genera el contexto propicio para el desarrollo de una actividad criminal con fines económicos como es el narcotráfico (Del Olmo 1992). Es decir, el tráfico ilícito de drogas es una actividad comercial en donde intervienen diversos recursos, tanto materiales como humanos, y se organiza de manera tal que cada actividad ofrece un valor al producto final.

La arquitectura organizativa del mercado ilegal de la droga se articula para introducir un servicio o mercancía ilegal a los espacios de consumo (Sarmiento \& Krauthausen 1991). El funcionamiento por compartimentos o nodos de modo descentralizado facilita el ciclo productivo de la droga a través de la adaptación del mercado ilegal, evitando que el negocio se vea afectado por la intervención del Estado (Kenney 2007), de modo que se busca aumentar los beneficios y minimizar la probabilidad de captura (Becker 2001, Rocha García 2001).

Los procesos de transformación, desde la instalación de los cultivos de coca hasta la producción de pasta básica de cocaína, (PBC) y cocaína se pueden dividir en actividades con objetivos específicos y en atención al perfeccionamiento del producto. En el VRAEM se identifican tres eslabones principales de la cadena de valor del narcotráfico: i) producción, ii) distribución y iii) comercialización. En cada uno de estos se busca producir un valor agregado al producto y se evita cualquier riesgo de control (Zevallos \& Mujica 2013).

En zonas productoras de hoja de coca como el Perú, también se realiza el refinamiento de pasta básica y clorhidrato de cocaína. La actividad en el VRAEM no se restringe al cultivo, sino que algunos de los que se iniciaron como agricultores dan espacio a las pozas de maceración y laboratorios de 
procesamiento (Zevallos \& Rojas 2012). Así, algunos campesinos cocaleros otorgan valor agregado a su producto y se han incorporado a la cadena de producción de droga (Méndez 2009). Además, el producto se transporta hasta los principales lugares para salir del valle cocalero. Los transportadores de droga son conocidos como «mochileros» $\mathrm{o}$ «cargachos» y trasladan los paquetes de drogas en pequeñas cantidades, regularmente por vía terrestre, desde las zonas de producción hacia los puntos de embarque, los «burriers» se encargan del traslado externo. De este modo, la atomización y fragmentación de las actividades del tráfico ilícito de drogas busca evadir la fiscalización del Estado.

En este escenario se emplean acciones para controlar el narcotráfico por diferentes actores. El trabajo policial que controla el tráfico ilícito de drogas en todas sus etapas (producción, distribución y comercialización) se organiza a partir de un Manual de Procedimientos (MAPRO) y anualmente la Dirección Antidrogas realiza un Plan Antidrogas (aprobado por el comando institucional y el director antidrogas). Además, las bases policiales descentralizadas desarrollan Planes de Operaciones que tienen como cimiento cumplir con los objetivos trazados en la Estrategia Nacional de Lucha Contra las drogas (20122016). Entonces, bajo estas premisas, se entiende que los agentes policiales en este ámbito tienen la capacidad burocrática de contar con habilidades técnicas, recursos y una organización determinada para aplicar en la resolución de complejas cuestiones de política pública (Rourke 1984), como hacer frente a la lucha contra las drogas mediante la interdicción de drogas.

No obstante, pese a que se han dispuesto planes operativos que detallan los procedimientos de intervención, en la aplicación de estos existen espacios de riesgo para transacciones irregulares o prácticas de corrupción. Estos espacios de riesgo son aquellos en donde la capacidad del Estado (representado por una entidad como la Policía Nacional) no garantiza control. Así, la capacidad estatal burocrática con la que formalmente cuentan los policías, puede tener limitaciones no solo por presiones competitivas externas, sino también debido a la forma real en que los funcionarios operan y se comportan dentro de su hábitat de trabajo (Rourke 1984). Un elemento importante es que no todos los espacios o situaciones de riesgo de corrupción a los que se enfrentan los policías son iguales y depende del tipo de actividad de la cadena de tráfico ilícito de drogas (TID). Así, por ejemplo, el cultivo de hoja de coca requiere de zonas de cultivo y zonas de acopio, en donde el control policial supone la fiscalización de la hoja de remisión que autoriza la posesión de hoja de coca, por ello, a este control se asocia como práctica de corrupción la autorización de toda aquella guía de remisión falsa o irregular. 
Para la reproducción de las actividades de refinamiento de derivados cocaínicos (tanto pasta como clorhidrato de cocaína), se requiere de insumos químicos como pozas y laboratorios de producción, de modo que la práctica de corrupción asociada a este espacio estaría relacionada con el soborno para omitir un certificado falso del Registro de Usuarios o también la posesión de bienes incautados en las pozas y/o laboratorios. En el caso del transporte, las medidas de control son distintas, se buscan los establecimientos de acopio y el trabajo policial se da en carreteras y pistas de aterrizaje clandestinas (PAC), por lo que las prácticas de corrupción serían las coordinaciones para la evasión de control en estos espacios.

Además, las prácticas de corrupción asociadas a las actividades de la cadena del TID cocaínico identificadas se configuran en los parámetros de una pequeña o mediana corrupción, por lo que, inicialmente, se puede indicar que las prácticas de corrupción se basan en contacto inmediato entre el actor vinculado al narcotráfico y el policía que encuentra una oportunidad de beneficiarse del contexto. Los tipos de prácticas de corrupción policial dependen de las oportunidades, que varían dentro de la institución y el ambiente de trabajo (Punch 2009). La siguiente tabla muestra las conductas policiales de corrupción adaptadas al contexto de interdicción de drogas en el VRAEM:

Tabla 2. Eslabones de la cadena del TID en el VRAEM: actividades y recursos

\begin{tabular}{|c|c|c|}
\hline Actividad & Espacios/Situación de riesgo & Prácticas \\
\hline \multirow[t]{2}{*}{$\begin{array}{l}\text { Cultivo de hoja de } \\
\text { coca }\end{array}$} & $\begin{array}{l}\text { Fiscalización de la hoja de remisión } \\
\text { que autoriza la posesión de hojas } \\
\text { de coca }\end{array}$ & $\begin{array}{l}\text { Autorización de documentos e } \\
\text { información falsificada }\end{array}$ \\
\hline & & $\begin{array}{l}\text { Sobornos para el tránsito del } \\
\text { cargamento }\end{array}$ \\
\hline \multirow[t]{2}{*}{$\begin{array}{l}\text { Refinamiento de } \\
\text { PBC y clorhidrato } \\
\text { de cocaína }\end{array}$} & $\begin{array}{c}\text { Comercialización de insumos } \\
\text { químicos }\end{array}$ & $\begin{array}{c}\text { Sobornos para la emisión de } \\
\text { certificación en el Registro de } \\
\text { Usuarios }\end{array}$ \\
\hline & $\begin{array}{l}\text { Zonas de producción (como pozas } \\
\text { y laboratorios) }\end{array}$ & $\begin{array}{l}\text { Posesión de bienes incautados en los } \\
\text { establecimientos de producción }\end{array}$ \\
\hline \multirow[t]{2}{*}{$\begin{array}{l}\text { Transporte interno } \\
\text { y externo }\end{array}$} & $\begin{array}{l}\text { Establecimiento de espacios } \\
\text { de acopio y venta }\end{array}$ & $\begin{array}{l}\text { Coordinación para evasión de } \\
\text { controles }\end{array}$ \\
\hline & $\begin{array}{c}\text { Carreteras y pistas de aterrizaje } \\
\text { clandestinas }\end{array}$ & $\begin{array}{c}\text { Coordinación para evasión de } \\
\text { controles }\end{array}$ \\
\hline
\end{tabular}

Fuente: Adaptado de los eslabones del TID citados por Zevallos \& Mujica (2014). 
En suma, las prácticas de corrupción están asociadas a los tipos de medidas de interdicción para el control de drogas. Es decir, ante la respuesta policial para controlar una actividad específica de cada eslabón de la cadena del TID en el VRAEM, el narcotráfico emplea un modo de evadir el control haciendo partícipe al policía, garantizando la continuidad del negocio ilícito. Además, de acuerdo a lo señalado, se trata principalmente de prácticas de pequeńa y mediana corrupción, en tanto no involucran grandes montos de dinero, vínculos con personas de altos cargos o responsabilidades, sino, por el contrario, con el funcionario local con quien se puede tener contacto directo. De este modo, los tipos de corrupción se relacionan con los niveles de organización burocrática policial y con el nivel operativo (Punch 2009), como en este caso que encontramos la adaptación de las funciones policiales operativas de interdicción de drogas en relación a las prácticas de corrupción.

\section{Medidas anticorrupción implementadas para las acciones de interdicción de drogas en el VRAEM}

En el plano policial, las principales acciones en materia anticorrupción se materializan en directivas y oficinas dentro de la institución con la finalidad de regular el comportamiento del policía en tanto funcionario público; es decir, sujetas a la labor administrativa, garantizan — principalmente - medidas de sanción. En función de hacer evidente el enfoque de las acciones anticorrupción, se consideran las medidas dispuestas en función de los actos de interdicción de drogas en el VRAEM.

En primer lugar, basadas en la organización jerárquica de la institución policial, para el control de prácticas de corrupción existen oficinas especializadas para el tratamiento de los casos denunciados, así como oficinas que - entre otras labores - regulan el comportamiento de su personal. Así, por lo menos cuatro entidades dentro de la Policía Nacional deben ponerse de acuerdo en la implementación: la Unidad Policial Local, la Inspectoría Regional del VRAEM, la Inspectoría General y la Dirección Contra la Corrupción (DIRCOCOR). Es decir, se cuenta con varios actores, de alcance nacional y local, que requieren coordinar sobre las actividades de control.

En principio, el servicio policial de interdicción de drogas responde a la organización interna de la Unidad Policial Local. Las labores determinadas en función de los objetivos pueden ser programados con anticipación o no; sin embargo, el reporte de las actividades diarias es una medida estipulada dentro del manual de procedimientos para las intervenciones antidrogas. Por ello, la irregularidad en los partes policiales, reporte de combustible y reporte de la 
droga incautada son elementos que serían sancionados como «Medida Muy Grave (MG)», ${ }^{4}$ para lo cual se determina el pase al retiro.

Seguidamente, cualquier irregularidad que suceda en las bases policiales debe ser atendida por la instancia inmediata superior, que en este caso corresponde a la Inspectoría Regional del VRAEM (IR-VRAEM). Así, la IR-VRAEM participa en el control de los operativos de interdicción en dos sentidos: controles inopinados y controles opinados. La oficina regional del VRAEM está ubicada en Huanta y las oficinas descentralizadas están en Kiteni y Pichari. Las denuncias de los casos irregulares detectados se formalizan en la oficina de Huanta. Luego, con la información recopilada al nivel mencionado, la Inspectoría General de la Policía Nacional, mediante el Tribunal Administrativo Disciplinario, está a cargo de la investigación disciplinaria ${ }^{5}$ y es la última instancia administrativa que declara un procedimiento disciplinario iniciado por infracciones Muy Graves declaradas en el artículo 42. DL N 1150.

De este modo, se cuenta con una tipificación legal que, en tanto determinación únicamente normativa, no constituye un mecanismo en sí sino un inventario de conductas con el propósito de ser sancionadas (Mujica 2011). Un primer problema, entonces, es que las medidas que existen no parecen responder a la configuración de un fenómeno de corrupción fragmentado, como se indicó en el capítulo anterior. Además, un detalle importante es que al buscar sancionar, se requiere tomar en cuenta la amplia dispersión de prácticas ya mencionadas previamente. Así, la amplia cantidad de pequeños casos de corrupción requieren de una importante capacidad de análisis y coordinación con los agentes entendidos en el tema, y sin ello, el sistema de control se sobrecarga en la revisión de la tipificación del delito en cada uno de los expedientes acumulados.

En cuanto a las medidas de prevención, estas son autogeneradas por los policías locales; por ejemplo, algunos jefes responsables de las bases policiales orientan a su personal de acuerdo a la formación personal. A diferencia de la sanción, las medidas de prevención son menos institucionalizadas. La

\footnotetext{
4 Según el Régimen Disciplinario de la PNP-DL No 1150, las infracciones son acciones u omisiones que atentan contra las obligaciones y deberes establecidos en la ley de la PNP, se clasifican en: Leves (L), Graves (G) y Muy Graves (MG).

5 La Comisión Especial de Reestructuración de la Policía Nacional, mediante informe aprobado por resolución suprema $\mathrm{N}^{\circ}$ 0200-2002-IN, recomendó la creación de la Oficina de Asuntos Internos, el fortalecimiento de la Inspectoría General de la PNP como subsistema autónomo a nivel nacional, y la reformulación del régimen disciplinario de la PNP con la formación del Tribunal Administrativo Disciplinario.
} 
Dirección Contra la Corrupción (DIRCOCOR) ${ }^{6}$ tiene como función el fortalecimiento del procedimiento de control y, a diferencia de las oficinas de inspectoría que investigan y sancionan, no cuenta con oficinas descentralizadas en el VRAEM sino solo a nivel de la región, y su intervención es para abordar casos de flagrancia.

\section{Tabla 3. Medidas anticorrupción implementadas para las acciones de interdicción de drogas}

\begin{tabular}{ccc}
\hline $\begin{array}{c}\text { Unidad Policial que } \\
\text { implementa }\end{array}$ & Medida anticorrupción & Tipo de medida \\
\hline Unidad Policial Local & Revisión de partes policiales & Combate $>$ Sanción \\
& Revisión del Combustible & Combate $>$ Sanción \\
& Revisión de almacén de droga & Combate $>$ Sanción \\
& Revisión del Servicio Policial & Combate $>$ Sanción \\
\hline $\begin{array}{c}\text { Inspectoría Regional } \\
\text { VRAEM }\end{array}$ & $\begin{array}{c}\text { Controles inopinado a las Unidades } \\
\text { Policiales en el VRAEM }\end{array}$ & Combate $>$ Sanción \\
& $\begin{array}{c}\text { Controles programados a las } \\
\text { Unidades Policiales }\end{array}$ & Combate -> Sanción \\
\hline $\begin{array}{c}\text { Inspectoría General } \\
\text { PNP }\end{array}$ & $\begin{array}{c}\text { Determinación en el Tribunal } \\
\text { de Disciplina Policial (última } \\
\text { instancia) }\end{array}$ & Combate -> Sanción \\
\hline $\begin{array}{c}\text { Dirección Contra } \\
\text { la Corrupción } \\
\text { (DIRCOCOR) }\end{array}$ & $\begin{array}{c}\text { Fortalecimiento de procedimientos } \\
\text { de control }\end{array}$ & Prevención \\
\hline
\end{tabular}

Fuente: Régimen de Disciplina Policial (DL N¹150), Inspectoría General y Dirección Contra la Corrupción de la Policía Nacional del Perú. Elaboración propia.

Por lo tanto, existe una concentración en medidas de sanción, poco eficaces frente a prácticas de pequeña corrupción. Así, por ejemplo, en relación a la sanción por pase al retiro por infracción Muy Grave, se encuentra que en materia de tráfico ilícito de drogas $(\mathrm{MG}-27)^{7}$ solo se registran dieciséis casos a nivel nacional en 2014 y de los cuales solo uno corresponde al VRAEM. ${ }^{8}$ Además, la concentración en medidas de sanción implica sobrecarga en las cuatro instancias de la PNP, generando problemas de coordinación; por ello,

\footnotetext{
6 La Dirección Contra la Corrupción (DIRCOCOR-PNP), Resolución Ministerial N 1544-2002-IN/PNP, es el órgano especializado de la Policía Nacional encargado de investigar y denunciar los delitos que concibe la corrupción, así como brinda el apoyo técnico y científico que requieren las autoridades del Poder Judicial y Ministerio Público

7 Infracción Muy Grave sobre TID (MG-27): «Participar, favorecer o facilitar actividades ilícitas relacionadas al tráfico ilícito de drogas, terrorismo, lavado de activos, secuestro, trata de personas, espionaje, robos, violación, extorsión y otros delitos graves" (Régimen de Disciplina Policial).

8 Sentencias del Tribunal de Disciplina Policial de la Inspectoría General de la PNP a junio de 2015.
} 
se cree que para lograr coordinar — por lo menos— se requieren de ciertas capacidades burocráticas.

\section{Limitaciones para la implementación de medidas anticorrupción en los operativos de interdicción de drogas en el VRAEM}

Tomando en cuenta los tipos de prácticas de corrupción en los operativos de interdicción de drogas y las medidas aplicadas para el control de estas prácticas, en esa sección atendemos las dificultades para implementarlas. Así, sumado a la incompatibilidad de las medidas anticorrupción con la situación real del fenómeno de corrupción, las medidas anticorrupción establecidas se enfrentan también a otras limitaciones de corte operativo, organizacional y logístico.

En función de lo mencionado, en el trabajo de campo como de gabinete se encontraron ejemplos concretos de limitaciones que se asocian a limitaciones burocráticas. Las principales limitaciones han sido tipificadas en relación a la ausencia o deficiencia de los elementos del diagnóstico organizacional de Oszlak (2006) como: 1) la disposición y gestión de recurso humano, que implica una cantidad insuficiente de personal y la poca especialización del mismo; 2) ineficacia administrativa, que es el procedimiento engorroso o la ausencia de dinamismos; 3) la disposición y gestión de logística, que sería el escaso presupuesto y la mala infraestructura; y 4) el factor organizativo, que responde a la descoordinación para el trabajo.

En cuanto a la disposición de gestión de recurso humano, los testimonios indican que es difícil controlar la corrupción cuando algunos policías toman su descanso y hacen uso de su autoridad para hacer intervenciones, apelando a su autoridad para obtener un beneficio de lo que han incautado. Por otro lado, en la otra situación, el personal de Inspectoría indica que es insuficiente y ello afecta a las investigaciones. En el VRAEM, la Inspectoría Regional se encuentra en Huanta, Ayacucho, en donde trabajan siete personas, en las oficinas descentralizadas de Kiteni trabajan cinco personas y en Pichari solo hay dos personas a cargo del control de los operativos de interdicción de su jurisdicción.

Del mismo modo, en la zona del VRAEM existen dos elementos que han dificultado el control de la corrupción: la capacitación y la especialización de los policías. Estos elementos se han debilitado mucho por la necesidad de contar con mayor personal cumpliendo con horas de trabajo y a disposición de cualquier eventualidad. El personal policial que atiende las investigaciones por corrupción tiene como conocimiento base el Régimen Disciplinario Policial, pero para el tratamiento de los casos denunciados también es necesario el 
conocimiento del derecho penal y la legislación en materia anticorrupción que no es implementada como una medida de capacitación desde la Policía Nacional. Por otro lado, la técnica en la pericia es una limitación proveniente de la especialización policial, la investigación y posterior resolución de los casos requiere de conocimiento especializado que permita usar bien herramientas como el parte policial, la entrevista a detenidos, la búsqueda de la prueba, etcétera, y todo ello se va perdiendo por desconocimiento o inexperiencia.

Respecto a la ineficacia administrativa, por ejemplo, la incorporación de otras labores en el servicio policial satura a los funcionarios en la atención de los casos que son investigados y descuida el control y monitoreo de las actividades en los operativos de interdicción y los recursos que se emplean en los mismos. La disposición de la gestión del recurso para el control de los operativos es ineficiente debido a que el mismo personal policial asignado tiene que investigar, capturar, hacer partes; los policías logran hacerlo en diez días y hasta que responde la fiscalía, hay que añadir cinco días más.

Del mismo modo, la disposición y gestión del recurso logístico en la zona del VRAEM dificulta el control de los operativos de interdicción de droga dado que son escasos los recursos para movilizarse en la zona. En la mayoría de unidades (tanto en las que operan en los operativos como en las que controlan el trabajo policial) se cuenta con un solo vehículo para todas las actividades de la Unidad Policial. El archivo documental también es necesario para constatar las irregularidades, no obstante, en varias oportunidades los funcionarios de control indican que la investigación no presenta datos contundentes y que ello también responde al uso de tecnología para demostrar la prueba, incluso en las intervenciones inopinadas se requiere el reactivo de tiocinato de cobalto, que no siempre es suministrado para las intervenciones.

En cuanto al factor organizativo, las acciones de interdicción están supeditadas a varios procesos que no provienen de la unidad de control descentralizada (la Inspectoría Regional VRAEM), sino de la Inspectoría General con sede en Lima. La coordinación con las Oficinas de Inspectoría de Kiteni y Pichari, aunque limitada, permitiría a la IR-VRAEM actuar prontamente, pero existe una dependencia administrativa para realizar las labores de control y por la cual no se puede operar a tiempo, otorgándole libertad al desarrollo de las irregularidades que suceden en un espacio y tiempo inmediato 
Tabla 4. Limitaciones para la implementación de las medidas anticorrupción

\begin{tabular}{|c|c|c|}
\hline Tipo de limitación & Descripción & Ejemplo \\
\hline \multirow[t]{2}{*}{$\begin{array}{c}\text { Disposición y } \\
\text { Gestión de RRHH }\end{array}$} & $\begin{array}{l}\text { Insuficiente personal. Poca } \\
\text { rotación. No especialización. }\end{array}$ & $\begin{array}{l}\text { En las oficinas descentralizadas: } \\
\text { Kiteni tiene } 5 \text { personas y en Pichari } 2 \\
\text { controlan su jurisdicción. }\end{array}$ \\
\hline & & $\begin{array}{l}\text { Poca especialización } \\
\text { en materia } \\
\text { anticorrupción y de } \\
\text { TID. }\end{array}$ \\
\hline $\begin{array}{c}\text { Ineficacia } \\
\text { administrativa }\end{array}$ & $\begin{array}{l}\text { Procedimiento engorroso. Ausencia } \\
\text { de dinamismo. }\end{array}$ & $\begin{array}{c}\text { El mismo personal policial asignado } \\
\text { tiene que investigar, capturar, hacer } \\
\text { partes, atestado policial, en } 15 \text { días: } \\
10 \text { días para el atestado policial, más } \\
5 \text { días de transporte al MP. }\end{array}$ \\
\hline $\begin{array}{l}\text { Disposición y gestión } \\
\text { de logística }\end{array}$ & $\begin{array}{l}\text { Escaso presupuesto. Mala } \\
\text { infraestructura. }\end{array}$ & $\begin{array}{c}\text { Infraestructura y condiciones de } \\
\text { almacén precario e inseguro: material } \\
\text { triplay. }\end{array}$ \\
\hline Factor organizativo & Descoordinación. & $\begin{array}{c}\text { Comunicación entre IR y las } \\
\text { inspectorías descentralizadas, entre IR } \\
\text { y IG, IG y DIRCOCOR. }\end{array}$ \\
\hline
\end{tabular}

Fuente: Adaptación del Diagnóstico organizacional de Oszlak (2006).

Elaboración propia.

En suma, las limitaciones relacionadas al aspecto burocrático tienen que ver con el funcionamiento, la organización y las competencias de los funcionarios policiales. Así, los elementos con los que gestiona la burocracia policial y las reglas de juego del aparato policial tienen efecto directo sobre la aplicación de las medidas anticorrupción en los operativos de interdicción de drogas y sobre la coordinación de los actores dentro de la Policía Nacional responsables de la implementación del control de estas prácticas anticorrupción. Esto es un elemento importante para ir aterrizando las limitaciones a nivel local, específicamente en los distritos que responden a la delimitación del VRAEM.

\section{Problemas burocráticos asociados al funcionario local para el control de la corrupción en los operativos de interdicción de drogas en el VRAEM \\ En el espacio más próximo a las actividades del narcotráfico, las limitaciones para el control de la corrupción hacen el escenario aún más complejo. En consideración del modelo "de abajo hacia arriba», los operadores locales del control, quienes deberían detectar e implementar las medidas anticorrupción en el espacio donde estas se configuran, presentan problemas asociados a 1) la}


posición del funcionario según su grado dentro de la jerarquía institucional y 2) la capacitación del funcionario policial para el desempeño de sus labores técnicas (Zuvanic, Iacoviello \& Rodríguez Gustá 2010).

Entonces, en estos planos se evidencia que la estabilidad laboral impacta en la implementación de las medidas anticorrupción en tanto el policía no tiene claro cómo desempeñar su labor, dado que en su formación académica no se ha contemplado en detalle el tipo de labor a la que se enfrentaría según las necesidades de la oficina a donde ha sido asignado. Por ejemplo, las unidades especiales seleccionan a su personal, y aquella rigurosidad, por dificultades de recursos y dedicación, no sucede a nivel nacional, ni siquiera con la totalidad de funcionarios policiales destacados al VRAEM. Normalmente, las medidas de prevención son aplicadas a los «grupos de élite» que van a trabajar operativos antidrogas específicos.

En este panorama, los oficiales PNP que están destacados en las inspectorías descentralizadas y son jefes responsables de los suboficiales, responden a su labor pero supeditados a los cambios de su cargo. Los oficiales, a quienes se les da la responsabilidad de control de la investigación de los casos de corrupción, normalmente permanecen hasta un periodo máximo de dos años en el cargo y si bien la institución policial tampoco les brinda capacitación especializada en esta materia, su experiencia y capacitación previa complementa su servicio. Aquellos policías que pertenecen a las unidades especializadas en materia de TID y están destacados en la zona del VRAEM facilitan el control y monitoreo de las labores de interdicción, pero este personal realiza la labor operativa, no la labor administrativa y de investigación que se ha asignado específicamente a la inspectoría. Es decir, los suboficiales que permanecen mayor tiempo en las inspectoría tienen baja capacitación en materia de TID y aquellos oficiales que tienen experiencia en materia de TID no pertenecen a las inspectorías descentralizadas porque su labor es más operativa.

Es decir, el equilibrio entre estabilidad o rotación del personal y el nivel de capacitación del mismo difiere entre las unidades especializadas en materia de TID (como los policías que pertenecen a la Dirección Antidrogas con base en el VRAEM) y las unidades de control (como las inspectorías descentralizadas del VRAEM). 
Tabla 5. Problemas burocráticos asociados al funcionario local

\begin{tabular}{lcc}
\hline $\begin{array}{c}\text { Problemas } \\
\text { burocráticos }\end{array}$ & Limitaciones asociadas & Ejemplo \\
\hline Estabilidad Laboral & $\begin{array}{c}\text { Posición del funcionario en } \\
\text { el cargo }\end{array}$ & $\begin{array}{c}\text { DIREJANDRO crea «grupos de élite» para } \\
\text { contar con personal especializado pero no es } \\
\text { permanente en la zona. }\end{array}$ \\
& $\begin{array}{c}\text { Rotación de oficiales supeditada al comando } \\
\text { policial. }\end{array}$ \\
\hline Capacitación Técnica & Conocimiento de la labor & $\begin{array}{c}\text { Suboficiales tienen mayor tiempo en bases } \\
\text { policiales pero sin especialización en TID ni } \\
\text { corrupción. }\end{array}$ \\
& & $\begin{array}{c}\text { La especialización en materia anticorrupción } \\
\text { y sobre TID está condicionada a: la } \\
\text { educación en escuelas a nivel subnacional. }\end{array}$ \\
\hline
\end{tabular}

Fuente: Adaptación de Zuvanic, Iacoviello, \& Rodríguez Gustá (2010).

Elaboración propia.

Estos elementos hacen difícil que se conozca qué se puede hacer para el control de la corrupción en caso de narcotráfico y, aun cuando se conozca, no se desempeñará adecuadamente en tanto ese personal sea reubicado indistintamente. Por lo tanto, el trabajo de los funcionarios locales también presenta limitaciones, asociadas a la labor del personal. Eso hace aún más difícil la implementación local de las medidas anticorrupción.

\section{CONCLUSIONES: CAPACIDADES BUROCRÁTICAS EN RESPUESTA A UN FENÓMENO SUBNACIONAL}

En lo planteado en este artículo encontramos importantes evidencias del tipo de limitaciones para la implementación de medidas anticorrupción en la interdicción de drogas en el VRAEM. De acuerdo a lo descrito, no solo tenemos una disociación entre el tipo de medida y el tipo de prácticas de corrupción que existen, sino también hay una sobrecarga y demora en el sistema al priorizar la sanción como medida de control, y problemas burocráticos limitan la efectividad de dicho control. Al mismo tiempo, hay problemas en el propio operador local que dificulta la coordinación con el resto de actores en la Policía Nacional responsables del control. Por lo que esta suma de elementos explica cuáles son las dificultades que existen en la coordinación de esa capacidad a nivel local de respuesta para hacer frente a la corrupción en la interdicción de drogas.

Una primera conclusión es la disociación entre el tipo de medida y el tipo de práctica de corrupción. En la investigación entendemos que la manifestación fragmentada de la corrupción en las acciones de interdicción es relevante 
para entender el diseño de la medida anticorrupción. Como hemos visto, las prácticas responden al tipo de riesgo y cada tipo de riesgo responde a un tipo de actividad. Esto está relacionado con lo discutido a partir de Mujica (2011), pues no hablamos de un fenómeno centralizado sino de entender cómo estas prácticas de corrupción se disponen en la realidad.

Como segunda conclusión, las medidas anticorrupción no responden al fenómeno de corrupción realmente existente para el caso del VRAEM. Priman las sanciones y son poco efectivas porque están pensadas en sancionar un fenómeno muy extendido, aun cuando las prácticas que identificamos responden a una estructura de pequeña y mediana corrupción, debido a su expansión. Puntualmente, en este caso de estudio, los actos de corrupción se caracterizan por actores que ocupan mandos administrativos y/o funcionarios de mandos bajos que interactúan con ciudadanos. Es decir, se trata de una "corrupción burocrática», propia de la administración pública en la implementación de políticas (Alfiler 1979).

La tercera conclusión es que además de la disociación mencionada, estas mismas medidas se enfrentan también a otras limitaciones de corte operativo, organizacional y logístico. Se trata de limitaciones de corte burocrático que hacen más difícil sostener el trabajo de sanción de prácticas de corrupción que actualmente emplea la policía. De acuerdo con Rourke (1984), las limitaciones a la burocracia no solo surgen de presiones externas, sino también de factores relacionados con el modo en el que las organizaciones operan y los burócratas se comportan; en ese sentido, el desempeño del policía para el control de la corrupción también depende del funcionamiento de la institución policial: los procedimientos internos, la organización, el régimen de disciplina, competencias policiales, entre otros.

Respecto a la cuarta conclusión, el elemento a manera de explicación de estas limitaciones, ya pensando específicamente en el operador local, radica en dos tipos de problemas burocráticos en los implementadores: estabilidad laboral y capacitación. Estos dos elementos hacen muy difícil que el operador local pueda vincularse a la coordinación con el resto de actores a distinto nivel institucional y esto impacta en la productividad del funcionario. En el VRAEM coexisten diversas unidades de la Policía Nacional, pero todos los policías trabajan en el mismo contexto de riesgo aunque en condiciones distintas y, precisamente, el empleo de los recursos requiere de competencias, habilidades y ubicación del funcionario en un cargo donde pueda llevarlas a cabo (Zuvanic, Iacoviello \& Rodríguez Gustá 2010). 
Como quinta conclusión, el policía del VRAEM, en tanto funcionario público en un espacio permeado por el tráfico ilícito de drogas, debe reconfigurar su trabajo al contexto político-administrativo mencionado y adecuarse a las circunstancias que este le permita (Lipsky 1971; Aguilar 1993). De este modo, para efectos de esta investigación, las medidas de control de la corrupción en un espacio subnacional necesitan enfocarse "de abajo hacia arriba», y es en ese sentido que pueden conseguir efectividad en el tratamiento de un caso de corrupción.

Entonces, la pertinencia del concepto «capacidades estatales» para este caso de estudio es poder comprender la suficiencia de la Policía — en representación del Estado- para, como indica Sikkink (1993), instrumentar los objetivos que este se propone. En los operativos de interdicción de drogas se pone a prueba lo que efectivamente hace y puede hacer la Policía; es decir, es la entidad burocrática que materializa al Estado (Oszlak 2006). Sin embargo, es importante precisar que la aproximación al componente burocrático no excluye los elementos políticos naturales de la institución policial, por lo que las relaciones interinstitucionales, "reglas de juego» y el vínculo con la normativa (Oszlak \& Orellana 2001) trascienden en la implementación del control de la corrupción en los operativos de interdicción de drogas.

Los resultados de la coordinación entre la Inspectoría Regional, la Inspectoría del VRAEM y las oficinas de disciplina descentralizadas redundan en la respuesta del policía asignado a la unidad policial local en el VRAEM. Cuando el policía local requiere actuar de inmediato ante la sospecha o denuncia de un acto de corrupción, la intervención depende del recurso logístico y humano designado a partir de una resolución dispuesta por la Dirección General de la Policía Nacional; es decir, el lazo legal y organizacional de la Policía interviene en qué y cómo se ejecuta en la base local. Además, como hemos visto, la actuación en el espacio subnacional no solo se ve afectado en los insumos burocráticos como recurso logístico o humano, sino también por la rotación según el cargo del policía y el conocimiento para poder cumplir su labor.

En ese sentido, la capacidad burocrática en el control de la corrupción en los operativos de interdicción de drogas a nivel subnacional está asociada al tipo de medida que se adopta según la configuración local del fenómeno de corrupción. Esta investigación reflexiona sobre el tipo de medidas de control actualmente empleadas. La sanción como medida de control de la corrupción ha tenido un impacto poco efectivo porque la presencia de corrupción en el VRAEM no corresponde a los pocos casos investigados y sancionados reportados. Así, mientras la realidad exige la atención al funcionario local, es cuestionable que 
persista una sanción inoperante de persecución del delito que sobrecarga al sistema de control y no piensa en lo que necesita el funcionario policial local para intervenir en la realidad y lo que la realidad demanda al policía local.

Por todo ello, sería importante explorar mucho más la capacidad de respuesta para controlar la corrupción en un espacio subnacional, no solo de la Policía sino de otros funcionarios locales que también participan en la lucha contra las drogas. No solo la Policía Nacional despliega un gran esfuerzo en este contexto, por lo que las limitaciones burocráticas no parecen ser de agencia y sería sustancial cuestionarse qué otros mecanismos emplea el Estado para llegar al nivel subnacional, cómo coordinan los distintos funcionarios públicos para combatir la corrupción en el VRAEM, cómo se disponen los controles para conducir procesos administrativos e incluso penales, dado los espacios de riesgo generados por el mercado ilícito. Estas y otras reflexiones introducen perspectivas más claras para la implementación efectiva de políticas subnacionales para el control de la corrupción frente a distintos ilícitos.

\section{Bibliografía}

Aguilar, L. F. (1993). «Estudio introductorio». En Aguilar L. F. La Implementación de las Políticas. México D.F.: Miguel Ángel Porrúa, pp. 14-91.

Alfiler, M. (1979). "Administrative Measures Against Bureaucratic Corruption: The Philippine Experience». Philippine Journal of Public Administration. 23, pp. 321-349.

Alonso, G. (2007). «Elementos para el análisis de las capacidades estatales». En Alonso, G. Capacidades estatales, instituciones y politica social. Buenos Aires: Prometeo Libros.

Baker, T \& J. B. Roebuck (1973). An empirical Typology of Police Corruption. Springfield, Il: Thomas. Barzelay, M. (2001). Atravesando la Burocracia. Una nueva perspectiva de la administración pública. México: Fondo de Cultura Económica.

Becker, G. (2001). «Crime and Punishment: An Economic Approach». Journal of Political Economy. Bersch, K.; S. Praça \& M. Taylor (2013). «State Capacity and Bureaucratic Autonomy Within National States: Maping the Archipielago of Excellence in Brazil». Presented at The Latin American Studies Association Conference.

Canache, D. \& M. Allison (2005). «Perceptions of Political Corruption in Latin American Democracies». Latin American Politics and Society. 47 (3), pp. 91-111. https:/doi.org/10.1353/lap.2005.0031

Colazingari, S. \& S. Rose-Ackerman (1998). «Explaining Corruption in a Paternalistic Democracy: Lessons from Italy for America». Political Sciencie Quarterly. 113 (3), pp. 447-470. https:/doi.org/10.2307/2658076

Cortázar, J. (2007). «Una mirada estratégica y gerencial de la implementación de los programas sociales». En Cortázar, J. Entre el diseño y la evaluación. El papel crucial de la implementación de los programas sociales. Washington: BID. 
Del Olmo, R. (1992). ¿Prohibir o domesticar? Politicas de drogas en América Latina. Caracas: Nueva Sociedad.

Doig, A. \& R. Theobald (2000). Corruption and Democratisation. London: Frank Cass. https://doi.org/10.4324/9781315039602

Hill, M., \& M. James (1993). The Policy Process in the modern capitalist state. New York: Harvester Wheatsheaf.

Hope, K. (2000). «Corruption and developmet in Africa». In HOPE, Kempe. Corruption and Development in Africa. Lessons from Country Case-Studies. New York: St. Martin's Press, pp. 17-39.

IDEI (2009). El Mapa del Narcotráfico en el Perú. Lima: Pontificia Universidad Católica del Perú.

Ivkovic, S. K. (2005). «Police (mis)behavior: A cross-cultural study of corruption seriousness». Policing. 28, pp. 546-566.

https:/doi.org/10.1108/13639510510614609

Jacob, Charles (1966). "Policy and Bureaucracy». New Jersey, DV Nostrand Co

Johnston, M. (1996). «The seach for definitions: The viability of politics and the issue of corruption». International Social Science Journal. N¹49, pp. 639-658.

Kenney, M. (2007). From Pablo to Osama: Trafficking and Terrorist Networks, Government Bureaucracies and Competitive Adaptation. Pennsylvania State University Press.

Lapalombara, J. (1994). «Structural and Institutional Aspects of Corruption». Social Research. 62 (2), pp. 325-351.

Lattuada, M. \& M. Nogueira (2011). «Capacidades estatales y políticas públicas. Una propuesta para el abordaje de las políticas agropecuarias en la Argentina contemporánea (1991 - 2011)». Estudios Rurales. Publicación de Centro de Estudios de la Argentina. Vol. 1, Nro. 1. Universidad Nacional de Quilmes.

http://ppct.caicyt.gov.ar/index.php/estudios-rurales/article/view/637

Lipsky, M. (1971). "Street-Level Bureaucracy and the Analysis of Urban Reform». Urban Affairs Quarterly. VI (4), pp. 391-409. https:/doi.org/10.1177/107808747100600401

Manor, J. (1999). The Political Economy of Democratic Decentralization. Washington D.C.: World Bank. https:/doi.org/10.1596/0-8213-4470-6

Matland, R. E. (1995). «Synthesizing the Implementation Literature: The Ambiguity-Conflict Model of Policy Implementation». Journal of Public Administration Research and Theory. J-, 146.

Mauro, P. (1995). "Corruption and growth». Quarterly Journal of Economics. CX/3, pp. 681-712. https:/doi.org/10.2307/2946696C

Melville Dalton (1959). «Men Wha Manage». Nova York, John Wiley \& Sons, Inc.

Méndez, M. (2009). El impacto del narcotráfico en el ámbito politico. La cadena narco: amenaza para la democracia y la gobernabilidad. En IDEI. El Mapa del Narcotráfico en el Perú. Lima: IDEI/ PUCP, pp. 43-58.

Moody-Stuart, G. (1997). Grand Corruption. Oxford: World View Publishing.

Mujica, J. (2011). Micropolíticas de la corrupción: Redes de Poder y Corrupción en el Palacio de Justicia. Lima: Asamblea Nacional de Rectores.

Nye, J. (1967). "Corruption and Political Development: A Cost-Benefit Analysis». American Political Science Review. 61 (2), pp. 417-427. https:/doi.org/10.2307/1953254

Oetiic (s/f). Oficina de Ética de la Investigación e Integridad Científica. PUCP.

http://vicerrectorado.pucp.edu.pe/investigacion/comite-de-etica/ 
Oszlak, O., \& E. Orellana (2001). El análisis de la capacidad institucional: aplicación de la metodología SADCI. Buenos Aires: Mimeo.

Oszlak, O. (2006). «Burocracia Estatal: política y políticas pública». POSTData, Revista de Reflexión y Análisis Político. Abril, 2006.

Perlman, B. (1989). Modernizing the Public Service in Latin America: Paradozes of Latin American Public Administration. International Journal of Public Administration. 12:4 (3), pp. 674-705. https:/doi.org/10.1080/01900698908524645

Perez, D. W. \& J.A. Moore (2002). Police ethics: A matter of character. Incline Village, NV: Copperhouse.

Peters, Guy (1999). "La Politica de la Burocracia». México DF, Fondo de Cultura Económica.

Punch, M. (2009). Police Corruption: deviance, reform and accountability in policing. New York: Taylor \& Francis Group.

Repetto, F. (2004). Capacidad estatal: requisito para el mejoramiento de la política social en América Latina. Documento de Trabajo I-52. Washington, D.C.: INDES.

Rocha García, R. (2001). «Antecedentes y Perspectivas del Narcotráfico en Colombia: Una mirada a las Políticas». Problemas del Desarrollo. Vol. 32, núm. 126.

Rose-Ackerman, S. (2001). La corrupción y los gobiernos: Causas, consecuencias y reforma. Madrid: Siglo XXI.

Roebuck, J. B. \& T. Barker (1974). «A typology of police corruption». Social Problems. 21, pp. 423-437. https:/doi.org/10.2307/799909

Rourke, F. (1984). Bureaucracy, politics and public policy. Tercera edición. Boston: Little Brown.

Sampford, C. (ed.) (2006). Measuring Corruption. Londres: Ashgate Publishing.

Sarmiento, L. \& C. Krauthausen (1991). Cocaina \& Co.: un mercado ilegal por dentro. Bogotá: Tercer Mundo.

Seligson, M. (2002). «The Impact of Corruption on Regime Legitimacy: A Comparative Study of Four Latin American Countries». Journal of Politics. 64 (2), pp. 408-433. https:/doi.org/10.1111/1468-2508.00132

Shleifer, A. \& R. Vishny (1993). «Corruption». The Quarterly Journal of Economics. 108, pp. 599-617.

https:/doi.org/10.2307/2118402

Sikkink, K. (1993). «Las capacidades y la autonomía del Estado en Brasil y Argentina. Un enfoque neoinstitucionalista». Desarrollo Económico. Volumen 32, número 128. https://doi.org/10.2307/3467177

Theobald, R. (1999). «So What Really is The Problem about Corruption?» Third World Quarterly. 20(3), pp. 491-502.

https:/doi.org/10.1080/01436599913640

Treisman, D. (2000). "The Causes of Corruption: A Cross-National Study». Journal of Public Economics. 76 (3), pp. 399-457.

https:/doi.org/10.1016/S0047-2727(99)00092-4

UNODC. (2014). Monitoreo de Cultivo de Hoja de coca en el Perú. Lima: Biblioteca Nacional del Perú.

Van Meter, D. \& C. Van Horn (1975). «The Policy Implementation Process: A conceptual Framework». Administration and Society. 6:4, pp. 445-488. https:/doi.org/10.1177/009539977500600404

Weber, Max (1946). “Bureaucracy”, en From Max Weber, H. H. Gerth y C. Wright Milis (comps.). Nueva York, Oxford University Press. 
Weyland, K. (1998). «The Politics of Corruption in Latin America». Journal of Democracy. 9:2, pp. 108-121.

https:/doi.org/10.1353/jod.1998.0034

Wilson, J. (1989a). «Corruption: The Shame of the States». In Heidenheimer et al. (eds.). P. C. handbook. New Brunswick/London: Transaction Publishers.

Zevallos, N., \& M. Rojas (2012). «Una lectura desde el Capital Política a la Estrategia de Seguridad en el VRAE». Politai. Vol. 3, No. 4.

Zevallos, N., \& Mujica, J. (2013). «Consideraciones sobre las tensiones en la intermediación Política en el Movimiento Cocalero en el Perú». Debates en Sociología. Nro. 38.

Zuvanic, L., Iacoviello, M., \& Rodríguez Gustá, A. (2010). «The Weakest Link: The Bureaucracy and Civil Service Systems in Latin America». In C. Scartascini, E. Stein, \& M. Tommasi. How Democracy Works: Political Institutions, Actors, and Arenas in Latin American Policymaking. Washington, D.C.: David Rockefeller Center for Latin American Studies and Harvard University, pp. 147-176. 